\title{
The oscillatory entrainment of virtual pitch perception
}

\author{
Aleksandar Aksentijevic ${ }^{1}{ }^{*}$, Anthony Northeast ${ }^{2}$, Daniel Canty ${ }^{3}$ and Mark A. Elliott ${ }^{3}$ \\ ' Department of Psychology, University of Roehampton, London, UK \\ ${ }^{2}$ Department of Psychology, University of Surrey, Surrey, UK \\ ${ }^{3}$ School of Psychology, National University of Ireland, Galway, Ireland
}

Edited by:

Robert J. Zatorre, McGill University, Canada

Reviewed by:

Tobias Overath, University College

London, UK

Robert J. Ellis, Beth Israel Deaconess

Medical Center, USA

\section{${ }^{*}$ Correspondence:}

Aleksandar Aksentijevic, Department of Psychology, Whitelands College,

University of Roehampton,

Holybourne Avenue, London

SW154JD, UK.

e-mail: a.aksentijevic@roehampton. ac.uk
Evidence suggests that synchronized brain oscillations in the low gamma range (around $33 \mathrm{~Hz}$ ) are involved in the perceptual integration of harmonic complex tones. This process involves the binding of harmonic components into "harmonic templates" - neural structures responsible for pitch coding in the brain. We investigated the hypothesis that oscillatory harmonic binding promotes a change in pitch perception style from spectral (frequency) to virtual (relational). Using oscillatory priming we asked 24 participants to judge as rapidly as possible, the direction of an ambiguous target with ascending spectral and descending virtual contour. They made significantly more virtual responses when primed at 29,31 , and $33 \mathrm{~Hz}$ and when the first target tone was harmonically related to the prime, suggesting that neural synchronization in the low gamma range could facilitate a shift toward virtual pitch processing.

Keywords: gamma-band, virtual pitch, pitch perception, pitch coding, harmonic templates, oscillatory priming

\section{INTRODUCTION}

Processing the pitch of harmonic complex tones represents one of the most important research topics in auditory perception and cognitive neuroscience (e.g., Patterson et al., 2002; Penagos et al., 2004; Micheyl and Oxenham, 2007; McLachlan, 2011). There is accumulating evidence that the perceptual integration of disparate harmonic components in primary auditory cortex (PAC) involves the formation of harmonic templates (Brunstrom and Roberts, 1998; Lin and Hartmann, 1998; Shamma and Klein, 2000; Bendor, 2011), that is, networks of medium-range connections between topographically non-adjacent neural loci which code harmonically related frequencies (e.g., Kadia and Wang, 2003). One issue of particular concern is the mechanism which mediates the transformation of spectral (frequency) information into "virtual" Gestalt-like pitch percepts (Terhardt, 1974, 1989). The "Gestaltness" of a virtual pitch percept is reflected in the fact that it arises only when the constituent components are related to each other in a specific way.

The fact that a single stimulus can provide two mutually exclusive forms of pitch information (spectral and virtual) offers a convenient way of investigating the dynamics of harmonic pitch integration. Typically, such stimuli consist of pairs of two-frequency tones (see Figure 1). The frequencies are selected so that the frequencies in the second tone are lower than those in the first. At the same time, the frequency distance between components increases in the second tone (Smoorenburg, 1970) producing two competing contours - a falling spectral (1A) and a rising virtual (1B) contour. The former is extracted from the frequencies of successive components and the latter is defined by the frequency of the missing fundamental - the inaudible first harmonic of a relevant series. The spectral mode relies on the computation of the frequency differences whereas the virtual mode involves abstraction of tonotopic relationships in the sense that harmonic distance becomes the new unit of information for the purposes of computation and comparison.

Studies using such ambiguous stimuli reveal considerable inter-individual differences in listening style. While some listeners are capable of hearing virtual pitch, others derive pitch information from the spectral contour (the frequency difference between successive harmonics) and this preference appears to be stable over time (Patel and Balaban, 2001). Moreover, the relationship between the two modes of pitch perception is unidirectional: Pantev et al. (2001) found that once trained to hear virtual pitch, listeners have difficulty reverting to the spectral listening mode (see also Deutsch and Steeger, 1978). According to Winkler et al. (1994), virtual pitch is coded in sensory memory and takes longer to process as compared to spectral pitch. While listeners can be described as adopting either a spectral or a virtual mode, repeated exposure to harmonic tones can lead to an irreversible switch from the former to the latter.

A recent investigation of harmonic pitch integration using a priming paradigm (Aksentijevic et al., 2011) suggests that priming in the low gamma range $(33 \mathrm{~Hz})$ is involved in the synchronization of templates involved in the coding of target frequencies harmonically related to the carrier frequency of the prime. The priming causes an "inharmonic pop-out," that is, an increased salience of targets that are not harmonically related to the prime. In a second study, (Aksentijevic and Elliott, under revision) the inharmonic pop-out was observed in participants with no musical training but not in musically trained participants. This suggests that prolonged exposure to harmonic input creates permanent connections between harmonic template components. Aksentijevic and Elliott argue that once these connections have been formed, 


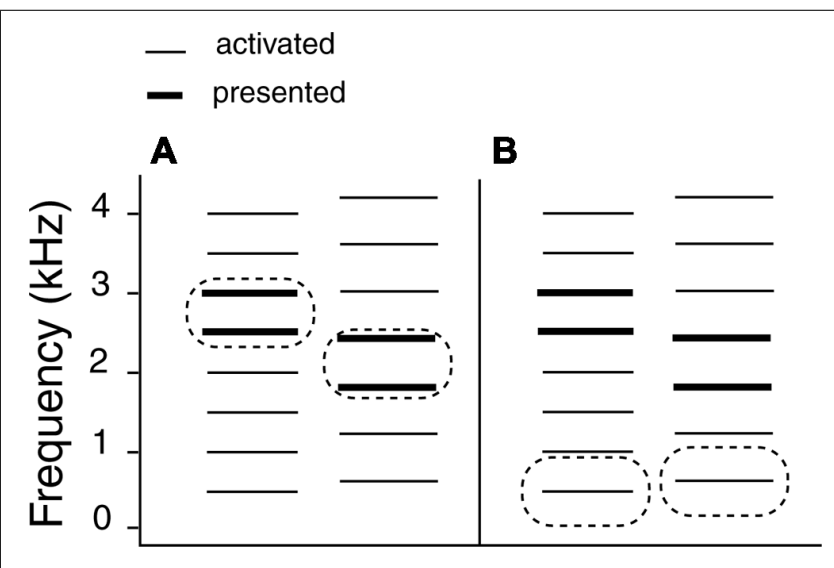

FIGURE 1 | Two modes of pitch perception. A pair of pure tones $(2500+3000 \mathrm{~Hz})$ is succeeded by another $(1800+2400 \mathrm{~Hz})$. The tone pairs activate harmonic templates of different fundamental frequencies (500 and $600 \mathrm{~Hz}$, respectively). (A) Spectral mode. A descending melodic contour is derived from the frequencies of audible components. (B) Virtual mode. An ascending contour is derived from the missing fundamental frequencies based on the mutual distance of individual harmonic components.

transient oscillatory synchronization becomes less relevant as a mechanism for binding.

Support for this view comes from a study by Schulte et al. (2002), in which listeners were exposed to an ambiguous melody over a period of several hours. The melody consisted of a sequence of pairs of pure tones which were arranged such that while their spectral contour produced a descending motif, the virtual melody was that of the well-known folk tune "Frère Jacques." After several hours of listening to the stimulus, all participants were able to recognize Frère Jacques. This shift to a virtual mode was accompanied by a functional reorganization of the PAC - by the anatomical redistribution of the evoked auditory gamma-band response (aGBR; e.g., Galambos, 1992). Following exposure, the aGBR ostensibly migrated away from the core toward the belt area in secondary auditory cortex suggesting that the shift to a virtual mode represents the consolidation of an oscillatory "harmonic memory" in the PAC (Jutras and Buffalo, 2010; Headley and Weinberger, 2011).

In the current study we employed ambiguous targets to investigate the hypothesis that rate-specific priming in the low gamma range (around $33 \mathrm{~Hz}$ ) would facilitate a perceptual shift toward the virtual listening mode. Spectral down - virtual up targets (SDVU) consisted of a pair of harmonics of the $500 \mathrm{~Hz}$ harmonic series $(2500+3000 \mathrm{~Hz})$ followed by a pair of harmonics of the $600 \mathrm{~Hz}$ series $(1800+2400 \mathrm{~Hz}$; see Figure 1). An SUVD target represented the reversed arrangement of the SDVU target. We conjectured a shift toward the virtual mode would be one consequence of the binding of components of a relevant harmonic template by means of oscillatory priming, which would promote the salience of the relational (virtual) pitch information relative to that carried by the spectral contour. In order to test this, we initially sought to replicate the original rate-specific priming effect found by Aksentijevic et al. (2011) at or around $33 \mathrm{~Hz}$.

In the original study, the binding effect was demonstrated indirectly - via the increased salience of inharmonic targets. Here, the two versions of the target stimulus permitted a direct test of the binding hypothesis. In the SUVD condition, the first target tone $(2500+3000 \mathrm{~Hz})$ is harmonically related to the prime. If the hypothesis that oscillatory priming affects only harmonic loci is correct, the oscillatory binding should facilitate virtual responses to SUVD targets which inherit the harmonic information entrained by the prime.

\section{MATERIALS AND METHODS PARTICIPANTS}

The study was approved by the Ethics Committee of the Department of Psychology, University of Roehampton, and conformed to the Helsinki Declaration on Human Rights. Twenty four undergraduate volunteers (five male) took part in the experiment (mean age 21.71 years, standard deviation 6.7 years, with one participant substantially older than the remaining participants). Two participants were left-handed and one was ambidextrous. Seven participants had received from 2 to 14 years of musical training. All participants reported normal hearing and none possessed absolute pitch. Apart from one drummer, all musically trained participants received training on pitched instruments including voice. All participants provided informed consent and received credits for taking part in the study.

\section{APPARATUS AND STIMULI}

Experimental trial and stimulus generation, as well as response timing were controlled by an IBM compatible computer with sound signals generated by an internal stereo sound card. Trial presentation and data collection were implemented using Superlab (v.4, Cedrus). The stimuli were presented diotically via Sennheiser HD 265 linear headphones.

Each trial consisted of two parts (see Figure 2). In the first, a $1000 \mathrm{~ms}$ tone-pip train (prime) was presented, designed so as to generate a gamma-band oscillatory steady state response (SSR; Galambos et al., 1981; Galambos and Makeig, 1992; Thut et al., 2011). Presentation rate was defined by the duration of individual tone pips. The prime was presented at 29, 31, 33, 35, or 37 pipsper-second (pps). The individual pips were created using $\mathrm{NCH}$ tone generator (Version 3.00; NCH Software) and were onsetand offset-ramped with ramps that were symmetrical and had a plateau of $33 \%$ of the overall period (Figure 1A). The trains were created using WavePad sound editor ( $\mathrm{NCH}$ Software).

The prime consisted of a repeating four-pip sequence, with every first pip carrying $1000 \mathrm{~Hz}$, and the remaining three pips carrying $500 \mathrm{~Hz}$. As demonstrated by Aksentijevic et al. (2011), this structure was critical in generating an internal oscillatory model responsible for the observed effects. Specifically, the regular reset of the oscillatory representation of the $500 \mathrm{~Hz}$ template by a $1000 \mathrm{~Hz}$ pip led to a periodic oscillatory model whose temporal properties could be examined very precisely. At 33 pps, the suprathreshold amplitude of the internal oscillatory model (expected to affect participants' responses) was predicted and confirmed at inter-stimulus intervals (ISIs) of 106 and $256 \mathrm{~ms}$ ( $\pm 5 \mathrm{~ms}$ ). Pip duration was a reciprocal of presentation rate (e.g., $30.3 \mathrm{~ms}$ at 33 pps). Following an ISI of either 100 or $400 \mathrm{~ms}$, a target was presented consisting of two $250 \mathrm{~ms}$ tones separated by an ISI of $100 \mathrm{~ms}$. In the original study, the priming effect was observed at 


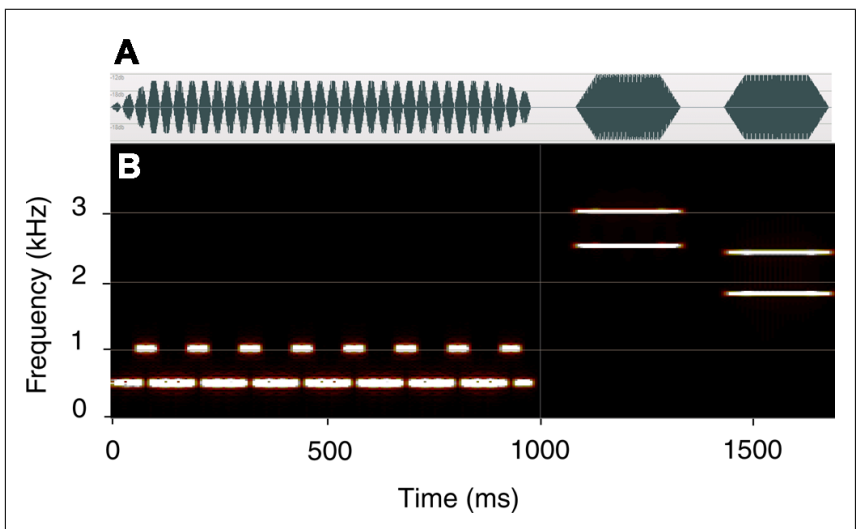

FIGURE 2 | Experimental trial. The prime is presented at the rate of $33 \mathrm{pps}$ and the ISI is $100 \mathrm{~ms}$. A spectral down - virtual up (SDVU) target is shown. (A) the waveform; (B) time-frequency plot. The first target tone carries the fifth and sixth harmonic of $500 \mathrm{~Hz}(2500+3000 \mathrm{~Hz})$ and is harmonically related to the prime. The second target tone carries the third and fourth harmonics of $600 \mathrm{~Hz}(1800+2400 \mathrm{~Hz})$. A reversed target (SUVD) was presented on $50 \%$ of the trials.

ISIs of 100 and $250 \mathrm{~ms}$. While we estimated the persistence of the oscillatory representation at about $400 \mathrm{~ms}$, we failed to observe any effects at ISIs above approximately $250 \mathrm{~ms}$. Consequently, the two ISIs were chosen a) in order to replicate the original finding of a rate-specific effect at $100 \mathrm{~ms}$ ISI and b) confirm the absence of the effect at longer intervals. Each target complex contained two frequencies and was symmetrically ramped (rise and fall time $50 \mathrm{~ms}$ ). The first tone carried 2500 and $3000 \mathrm{~Hz}$ (the fifth and sixth harmonics of the baseline prime frequency of $500 \mathrm{~Hz}$ ) while the second carried 1800 and $2400 \mathrm{~Hz}$ (the third and fourth harmonics of $600 \mathrm{~Hz}$ ). This produces two perceptual solutions. If listeners derive contour from the spectral information (frequency of successive harmonics), they hear a descending contour. Conversely, if they compute contour from the harmonic relationship between concurrent harmonics (virtual pitch), they hear an ascending contour. This target is labeled SDVU. It should also be noted that the first tone of the SDVU target was harmonically, and the second inharmonically, related to the prime. A reversed version of the target (spectral up - virtual down; SUVD) was presented on $50 \%$ of the trials in order to control for the harmonic relationship between the prime and target and increase stimulus variability. To recapitulate, the two target conditions were generated by inverting a single two-tone stimulus. Both versions of the target were presented an equal number of times.

\section{DESIGN}

The study employed a within-subjects design with factors ISI $(100 \mathrm{~ms}, 400 \mathrm{~ms})$, rate $(29,31,33,35,37 \mathrm{pps})$ and target direction (SDVU, SUVD). There were 10 trials per condition resulting in 200 trials per participant. Trials were presented pseudo-randomly for each participant across five blocks of 40 trials per block. Each session commenced with a 40 -trial practice block.

\section{PROCEDURE}

The experiment was conducted in a sound-attenuated experimental room. Stimulus intensity was held constant throughout [average $60 \mathrm{~dB}$ sound pressure level (SPL); A weighting; average error $2 \mathrm{~dB}$ SPL], examined using an artificial ear and an Adastra analog sound level meter, (Model 952.422, slow response), with the shape of the stimuli examined using WavePad sound editor. Each trial commenced with a $500 \mathrm{~ms}$ cross appearing at the center of the screen. Participants were instructed to focus on the target and indicate as quickly as possible if the target was going up or down by pressing the appropriate keyboard key with a finger of their dominant hand. In conjunction with target configuration, this task would produce response profiles corresponding to individual propensity to hear virtual pitch. Response time was measured from target onset and a new trial was initiated by the response. An individual session lasted approximately $15 \mathrm{~min}$.

\section{RESULTS}

Prior to analysis, 43 trials (out of 4800) were removed because they contained task-irrelevant responses. Data were analyzed in two stages. First, an omnibus analysis was performed on responsetime (RT) data in order to test against the results of Aksentijevic et al. (2011) and to establish the presence and frequency of a ratespecific priming effect in the current paradigm. For this, anti-logs of the means of the log-transformed RT distributions were subject to a three-way repeated-measures ANOVA with factors ISI (100, $400 \mathrm{~ms})$, rate $(29,31,33,35$, and $37 \mathrm{pps})$ and target direction (SDVU, SUVD). The main effect of target direction was significant $\left[F(1,23)=10.12, M S E=14432.1, p<.01 ; \eta_{p}^{2}=.31\right]$ with SDVU targets being registered more rapidly (mean difference $=39 \mathrm{~ms}, S E M=13 \mathrm{~ms}$ ). The three-way interaction was also significant $\left[F(4,92)=3.03, M S E=8011.66, p<.05 ; \eta_{p}^{2}=.12(\right.$ see Figure 3)], resulting from a significant difference in the effect of rate at the two ISIs as confirmed by the inspection of Bonferroniadjusted post-hoc comparisons. Specifically, at $100 \mathrm{~ms}$ ISI, there was a significant RT advantage for SDVU targets at 31 pps (difference: $64 \mathrm{~ms}, S E M=24.47, p<.05)$, while by contrast at $400 \mathrm{~ms}$ ISI, there was a large and significant $118 \mathrm{~ms}(S E M=25.82, p<.001)$ RT advantage for SDVU targets that followed a 33 pps prime consistent with the rate-specific inharmonic pop-out reported by Aksentijevic et al. (2011).

Our main hypothesis concerned the idea that gamma-band entrainment at specific rates would promote the shift toward virtual perceptual mode. In order to test this, participants' responses were coded with regard to their listening style. "Down" responses to SUVD targets and "Up" responses to SDVU targets were coded as "virtual". The opposite, reciprocal combination was coded as "spectral". Overall, the percentage of virtual responses was $49.7 \%$ (2362 out of 4757 responses), suggesting an equal representation of the two listening styles. Three participants made close to $100 \%$ virtual responses while three made less than $5 \%$ virtual responses. Four participants made an approximately equal number of spectral and virtual responses. This is consistent with the observation of a considerable inter-subject variability with respect to listening mode (e.g., Deutsch and Steeger, 1978; Patel and Balaban, 2001; Schneider et al., 2005) and suggests that the current task could be used as a convenient diagnostic test of listening style. Some musically trained participants produced more spectral responses suggesting that musical training does not automatically lead to a virtual shift. This is in broad agreement with the finding by 


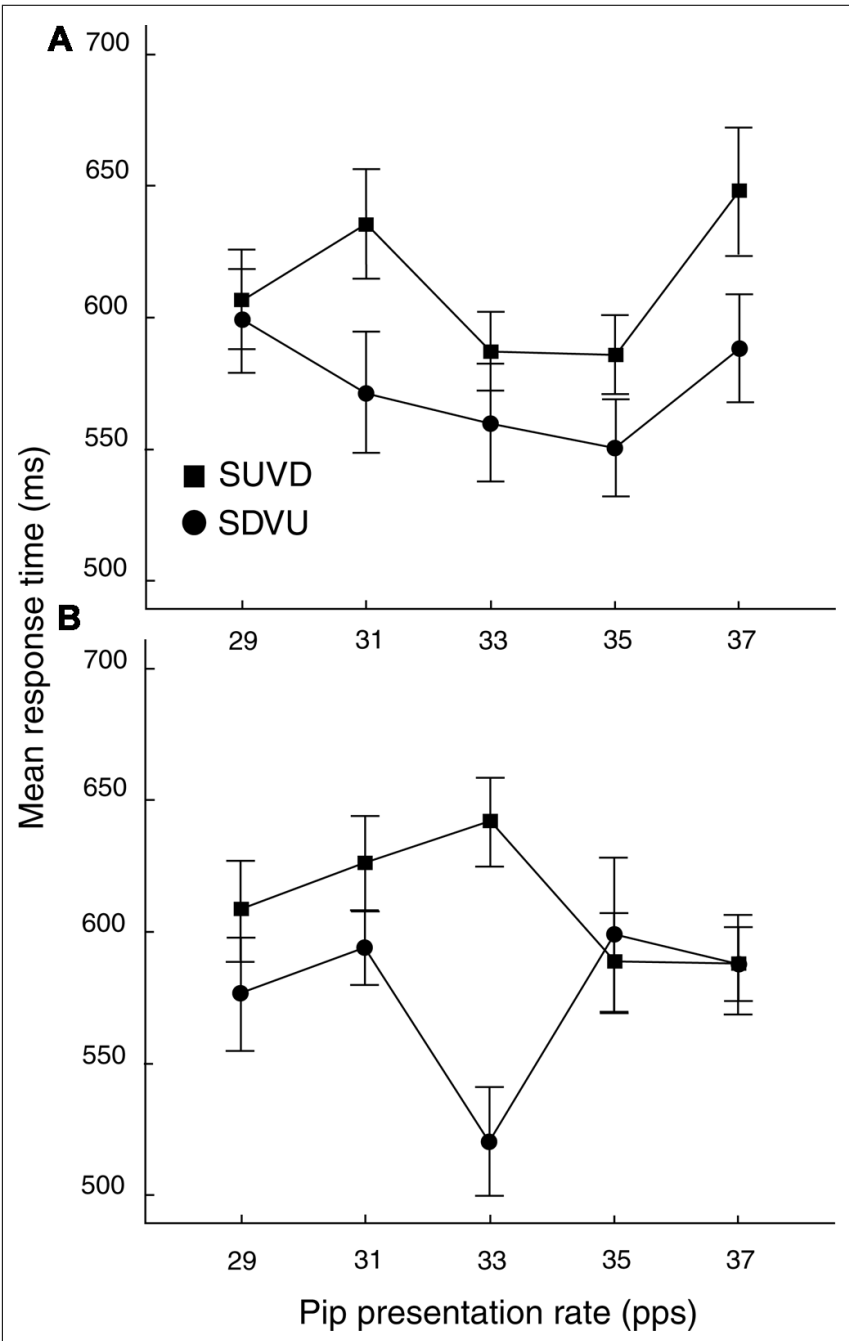

FIGURE 3 | Mean RTs ( \pm 1 SEM) as a function of ISI, rate and target. (A) Rate by target interaction at $100 \mathrm{~ms}$ ISI. (B) Rate by target type interaction at $400 \mathrm{~ms}$ ISI. SDVU = spectral down - virtual up; SUVD = spectral up - virtual down. SEM values were computed using the procedure described by Loftus and Masson (1994).

Schneider et al. (2005) of a bimodal distribution of listening style with the majority of listeners (mostly musicians), exhibiting a relatively strong preference for either style and some showing a mixed profile.

A three-way ANOVA with factors ISI (100, $400 \mathrm{~ms})$, rate (29, 31, 33, 35, 37 pps) and target direction (SDVU, SUVD) was performed on the percentages of virtual responses across all conditions (Figure 4). The main effect of target direction was significant $\left[F(1,23)=5.16, M S E=.137, p<.05 ; \eta_{p}^{2}=.18\right]$ indicating that more virtual responses were made to SDVU targets (mean difference $=6.8 \%, S E M=3.3 \%$ ). In addition, this effect was influenced by presentation rate as indicated by a significant rate by target direction interaction $[F(4,92)=3.57, M S E=.009, p<.01$; $\left.\eta_{p}^{2}=.13\right]$. Inspection with Bonferroni correction revealed this to be caused by a greater proportion of virtual responses to SDVU relative to SUVD targets at $29(11 \%, S E M=.04 \%, p<.01), 31$

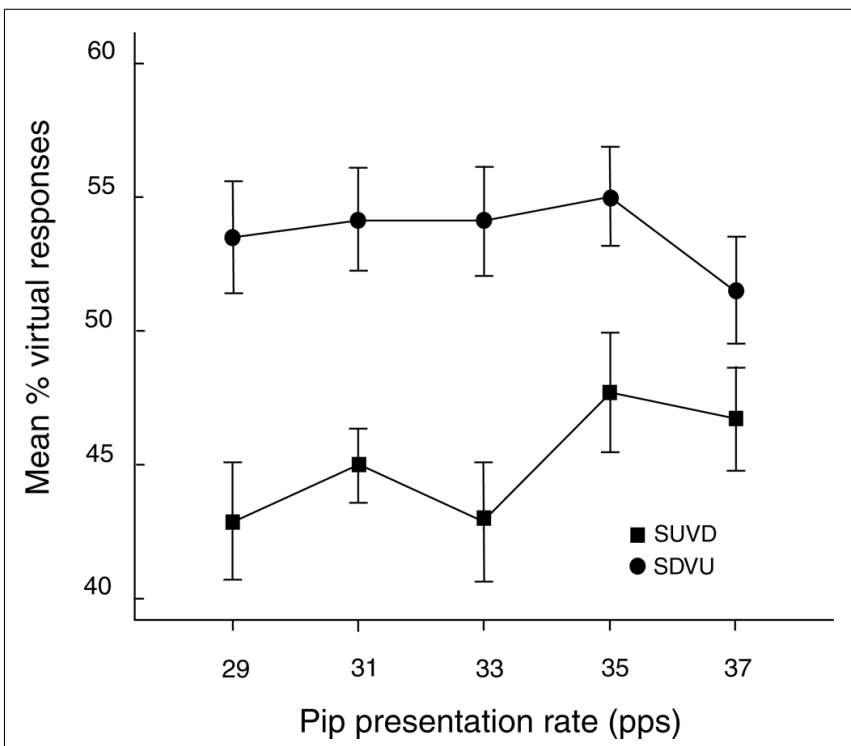

FIGURE 4 | Mean percentage ( \pm 1 SEM) of virtual responses as a function of rate and target. SDVU = spectral down - virtual up; SUVD $=$ spectral up - virtual down.

$(7 \%, S E M=.03 \%, p<.05)$ and 33 pps $(11 \%, S E M=.04 \%$, $p<.05)$ but not at higher rates.

To examine the relationship between RT performance and listening style, the RT ANOVA was rerun with listening style as a between-subject factor. "Listening style" was defined in terms of preponderance of virtual or spectral responses. The four listeners who had made between 50 and 55\% virtual or spectral responses were removed from analysis and the others were assigned to either a "virtual" or "spectral" condition. Apart from the obvious main effect of listening style $[F(1,18)=93.55, M S E=20.52$, $\mathrm{p}<.001$; $\left.\eta_{p}^{2}=.84\right]$, no significant effects of the latter factor were observed suggesting that the relationship between the two tasks was complex and would require further investigation. First, the interaction between listening style and RT facilitation might be observable only in "intermediate" listeners - those who are most likely to be affected by priming. We might not have had enough of these responders to observe the relationship between RTs and listening style and this justifies further experimental investigation. Second, the RT effects of priming might be at least partly independent of the virtual shift. To illustrate, there is evidence that virtual pitch perception requires more time relative to spectral pitch perception (e.g., Winkler et al., 1994). So, the RT effects could be confounded by the slowing down with respect to the virtual responses. It is worth noting that the same result was obtained with a stricter (75\%) exclusion criterion.

\section{DISCUSSION}

The current study investigated the effects of oscillatory gammaband priming (Aksentijevic et al., 2011) on the perceptual classification of ambiguous melodies. As shown in previous research (Schulte et al., 2002), a spectral perceptual mode is characterized by a focus on the frequency components that constitute complex tones. Conversely, virtual listening relies on the Gestalt properties 
of the stimulus, namely the relationships between harmonics. In most normal listening situations, this dichotomy is not relevant because complex tones (e.g., musical instrument tones or voice) are present in their entirety. In situations in which the two modes are brought into conflict, listeners need to make a decision based on the relative strength of either representation. In the present study, a two-tone stimulus was presented that could be interpreted either spectrally or virtually. Virtual responses were defined in reference to a specific target. This meant that response probabilities could not be established a priori (as would have been the case with a up/down responses to a binary independent variable) but depended on the listening style - individual propensity to hear spectral or virtual pitch. This meant that our results reflected the composition of the sample, that is, the presence of participants whose responses could be affected by priming. A sample comprising a large number of strong virtual or spectral listeners would likely not have produced significant effects.

The principal finding was that more virtual responses were made to SDVU relative to SUVD targets when the primes were presented at 29, 31, and 33 pps and this effect was independent of the interval between the prime and the target. The results suggest that the priming in the low gamma range could promote the binding of harmonically related components into a template thereby increasing the perceptual salience of the virtual Gestalt. The observed dependence on the type of target suggests that the presence of the first, harmonically related target tone played a role in the shift by re-establishing the entrained harmonic representation.

Figure 5 illustrates the relationship between harmonic templates activated by the stimuli. The left-hand side illustrates the SDVU condition. The prime $(500+1000 \mathrm{~Hz})$ activates the $500 \mathrm{~Hz}$ template, which in turn results in an oscillatory representation that persists following the prime offset (top left). The first target tone $(2500+3000 \mathrm{~Hz})$ fits into the active template helping to maintain the representation. The second tone $(1800+2400 \mathrm{~Hz})$ does not fit into the active template causing a reset in the oscillatory representation. This increases the salience of the conflicting $600 \mathrm{~Hz}$ template and facilitates the virtual "up" decision, but only at 29, 31, and 33 pps in broad agreement with Aksentijevic et al. (2011). The reinforcement of the oscillatory $500 \mathrm{~Hz}$ template representation by the first target tone contributes to the virtual shift. By contrast, the first tone in a SUVD target $(1800+2400 \mathrm{~Hz})$ would have disrupted the $500 \mathrm{~Hz}$ template entrained by the prime immediately leading to its re-establishment following the second tone. Since the response was based on comparing the second target tone to the first one, the time was not sufficient for the latter to establish an alternative harmonic representation, resulting in ambiguity. This in turn would have reduced the salience of the second target tone relative to the SDVU condition.

The absence of ISI effects in direction judgment data could be explained in terms of the difference between RT and direction aspects of the task. As shown by Aksentijevic et al. (2011), rate-specific RT effects were not mediated by consciousness (participants were unable to report the harmonicity relationship of the target to the prime). This is not the case with up-down decisions which require conscious control. While our results suggest that the processing advantage conferred by gamma priming benefits decisional processes, it is unlikely that this benefit derives directly from sensitivity to the temporal code of the entrainer. Instead, this code raises the value of a structural criterion in the stimulus that influences decisional processes. It is likely that decisions are affected by an increased salience of the virtual pitch representation, influenced in turn by the temporal parameters of the entrained oscillatory representation whose effects are ISI specific.

The importance of priming rate was corroborated by the timespecific RT enhancement mediated by target and ISI. The results indicate that SDVU targets were processed faster while producing

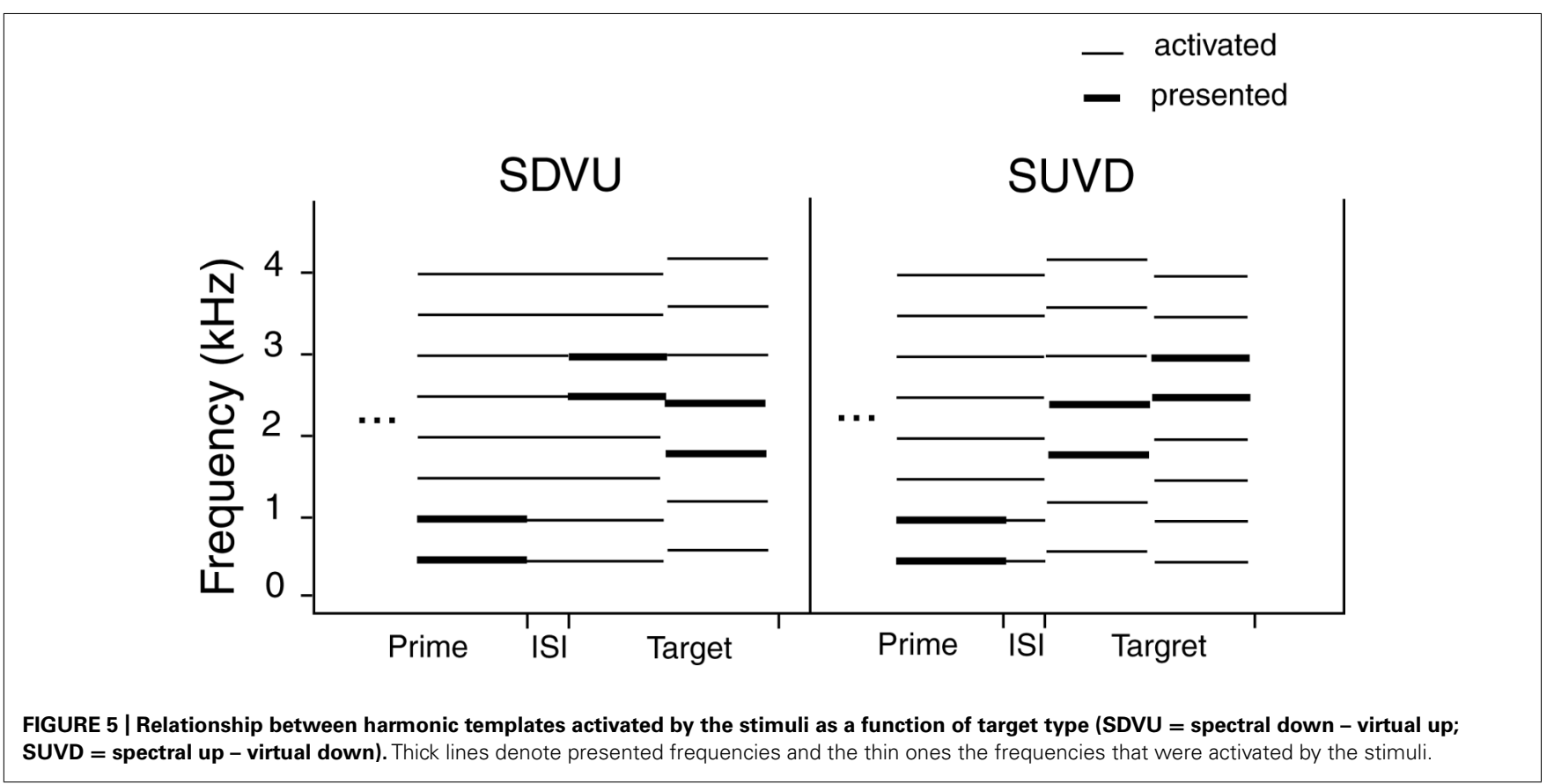


more virtual responses when primed in the low gamma range. At the shorter ISI of $100 \mathrm{~ms}$, responding was faster for SDVU targets if they were primed at 31 pps. At the longer ISI, the inharmonic advantage was observed at 33 pps. Given the temporal specificity of the stimuli, this is in agreement with previous findings of an inharmonic RT advantage for stimuli presented at 33 pps (Aksentijevic et al., 2011). Aksentijevic et al. (2011) reported reliable gammaband priming for ISIs of up to $250 \mathrm{~ms}$. The current unexpected finding of a rate-specific inharmonic facilitation at $400 \mathrm{~ms}$ ISI indicates that residual oscillatory activity in the low gamma range persists for longer than previously found and that it can be resynchronized by non-modulated targets. While in the original study, both the prime and the target consisted of tone-pips, here targets contained non-modulated sinusoids. Given the extreme temporal specificity of the effect $( \pm 2 \mathrm{~ms})$, a possible explanation is that the presence of the uninterrupted target sinusoid facilitates the resynchronization of the oscillatory representation. Nevertheless, further research is needed to elucidate the relationship between this late effect and the temporal parameters of the entrained oscillatory model.

The current findings of a rate-related shift in listening mode are consistent with an interaction between two (as of now putative) systems, namely, the pitch extraction system located in the PAC (lateral Heschl's gyrus; Schneider et al., 2005; Bendor and Wang, 2008, 2010) and the contour processing system located in the secondary auditory areas (planum temporale and planum polare; Puschmann et al., 2010). While contour can be extracted from spectral and virtual pitch information, it is hypothesized that the consolidation of harmonic templates underpinned by gamma oscillations leads to the change in the contour processing

\section{REFERENCES}

Aksentijevic, A., Barber, P. J., and Elliott, M. A. (2011). Process timing and its relation to the coding of tonal harmony. J. Exp. Psychol. Hum. Percept. Perform. 37, 1628-1642.

Bendor, D. (2011). Understanding how neural circuits measure pitch. J. Neurosci. 31, 3141-3142.

Bendor, D., and Wang, X. (2008). Neural response properties of primary, rostral, and rostrotemporal core fields in the auditory cortex of marmoset monkeys. J. Neurophysiol. 100 , 888-906.

Bendor, D., and Wang, X. (2010). Neural coding of periodicity in marmoset auditory cortex. J. Neurophysiol. 103, 1809-1822.

Brunstrom, J. M., and Roberts, B. (1998). Profiling the perceptual suppression of partials in periodic complex tones: further evidence for a harmonic template. J. Acoust. Soc. Am. 104, 3511-3519.

Deutsch, W., and Steeger, R. (1978). "Binaurales residuum versus lokalisation," Mitteilungen der Kommission für Schallforschung, Vol. 5 (Österreichische Akademie der Wissenschaften), 41-53.
Galambos, R. (1992). “A comparison of certain gamma-band $(40-\mathrm{Hz})$ brain rhythms in cat and man," in Induced rhythms in the brain, eds E. Basar, and T. M. Bullock (Boston: Birkhäuser), 201-216

Galambos, R., and Makeig, S (1992). Physiological studies of central masking in man. II: Tone-pip SSRs and the masking level difference. J. Acoust. Soc. Am. 92, 2691-2697.

Galambos, R., Makeig, S., and Talmachoff, P. J. (1981). A 40-Hz auditory potential recorded from the human scalp. Proc. Natl. Acad. Sci. U.S.A. 78, 2643-2647.

Headley, D. B., and Weinberger, N. M. (2011). Gamma-band activation predicts both associative memory and cortical plasticity. J. Neurosci. 31, 12748-12758.

Jutras, M. J., and Buffalo, E. A. (2010). Synchronous neural activity and memory formation. Curr. Opin. Neurobiol. 20, 150-155.

Kadia, S. C., and Wang, X. (2003). Spectral integration in the Al of awake primates: neurons with singleand multipeaked tuning characteristics. J. Neurophysiol. 89, 1603-1622.

system such that it switches to computing the contour information from the distances between individual harmonics (the pitch of the fundamental) rather than their position on the tonotopic axis.

\section{CONCLUSION}

The current study provides the first piece of behavioral evidence that synchronized brain oscillations in the low-gamma range could promote the shift toward the virtual listening mode. Our results might have important implications for pitch perception research in two ways. First, the current task appears to represent a simple and useful diagnostic of pitch processing style. Subject to validation, this could be applied in a wide variety of experimental and applied settings in which perception plays an important role (e.g., auditory and musical development, autism, musicianship, hemispheric lateralization). Second, our results suggest that changes in pitch processing can be effected rapidly, without prolonged exposure and/or training. A systematic multi-method investigation of the time course, persistence and reversibility of the observed priming effect would afford important insights into the temporal and spatial parameters of pitch processing.

At the same time, the current design in which virtual responses were reciprocals of spectral responses could not exclude the possibility that gamma priming somehow inhibited spectral responses. While this is not likely, further studies are needed to elucidate this issue. Future research will also seek to corroborate this evidence by combining behavioral and electroencephalography (EEG) data. It will also examine the role of the relationship between spectral and virtual frequencies in modulating the intensity and time course of this virtual shift.

Lin, J. Y., and Hartmann, W. M. (1998). The pitch of a mistuned harmonic: evidence for a template model. J. Acoust. Soc. Am. 103, 2608-2617.

Loftus, G. R., and Masson, M. E. J. (1994). Using confidence intervals in within-subject designs. Psychon. Bull. Rev. 1, 476-490.

McLachlan, N. M. (2011). A neurocognitive model of recognition and pitch segregation. J. Acoust. Soc. Am. 130, 2845-2854.

Micheyl, C., and Oxenham, A. J. (2007). Across-frequency pitch discrimination interference between complex tones containing resolved harmonics. J. Acoust. Soc. Am. 121, 1621-1631.

Pantev, C., Engelein, A., Candia, V., and Elbert, T. (2001). "Representational cortex in musicians: plastic alterations in response to musical practice," in The Biological Foundations of Music, eds R. J. Zattore, and I. Peretz (New York: The New York Academy of Sciences), 300-314.

Patel, A. D., and Balaban, E. (2001). Human pitch perception is reflected in the timing of stimulus-related cortical activity. Nat. Neurosci. 4 839-844.
Patterson, R. D., Uppenkamp, S., Johnsrude, I. S., and Griffiths, T. D. (2002). The processing of temporal pitch and melody information in auditory cortex. Neuron 36, 767-776.

Penagos, H., Melcher, J. R., and Oxenham, A. J. (2004). A neural representation of pitch salience in nonprimary human auditory cortex revealed with functional magnetic resonance imaging. J. Neurosci. 24, 6810-6815.

Puschmann, S., Uppenkamp, S., Kollmeier, B., and Thiel, C. M. (2010). Dichotic pitch processing activates centre in Heschl's gyrus. Neuroimage 49, 1641-1649.

Schneider, P., Sluming, V., Roberts, N., Scherg, M., Goebel, R., Specht, H. J., et al. (2005). Structural and functional asymmetry of lateral Heschl's gyrus reflects pitch perception preference. Nat. Neurosci. 8, 1241-1247.

Schulte, M., Knief, A., Seither-Preisler, A., and Pantev, C. (2002). Different modes of pitch perception and learning-induced neuronal plasticity of the human auditory cortex. Neural Plast. 9, 161-175. 
Shamma, S., and Klein, D. (2000). The case of the missing pitch templates: how harmonic templates emerge in the early auditory system. J. Acoust. Soc. Am. 107, 2631-2644.

Smoorenburg, G. F. (1970). Pitch perception of two-frequency stimuli. J. Acoust. Soc. Am. 4, 924-942.

Terhardt, E. (1974). Pitch, consonance, and harmony. J. Acoust. Soc. Am. 55, 1061-1069.

Terhardt, E. (1989). Warum hören wir Sinustöne? Nat. Sci. (Naturwissenschaften) 76, 496-504.
Thut, G., Schyns, P. G., and Gross, J. (2011). Entrainment of perceptually relevant brain oscillations by non-invasive rhythmic stimulation of the brain. Front. Psychol. 2:170. doi: 10.3389/fpsyg.2011.00170

Winkler, I., Tervaniemi, M., and Näätänen, R. (1994). Sensory memory for virtual pitch (Abstract). 7th IOP Scientific Meeting/International. J. Psychophysiol. 18, 157.

Conflict of Interest Statement: The authors declare that the research was conducted in the absence of any commercial or financial relationships that could be construed as a potential conflict of interest.

Received: 23 November 2012; accepted: 05 April 2013; published online: 25 Apri 2013.

Citation: Aksentijevic A, Northeast A, Elliott MA and Canty D (2013) The oscillatory entrainment of virtual pitch perception. Front. Psychol. 4:210. doi 10.3389/fpsyg.2013.00210
This article was submitted to Frontiers in Auditory Cognitive Neuroscience, a specialty of Frontiers in Psychology.

Copyright (C) 2013 Aksentijevic, Northeast, Elliott and Canty. This is an openaccess article distributed under the terms of the Creative Commons Attribution License, which permits use, distribution and reproduction in other forums, provided the original authors and source are credited and subject to any copyright notices concerning any third-party graphics etc 